\title{
Coronavirus (SARS-CoV-2) Cardiovascular Disease (CVD) Conditions Relationship in Diabetic and SCA Patients: a Review
}

\author{
Favour Deborah Adaugo Onyelowe ${ }^{1}$, Kennedy Onyelowe $^{2}$
}

${ }^{1}$ Ebonyi State University

P. M. B. 053, Abakaliki, Nigeria

${ }^{2}$ Kampala International University

Box 20000, Ggaba Road, Kansanga, Kampala, Uganda

DOI: $10.22178 /$ pos.60-3

LCC Subject Category: WC500-593

Received 26.06.2020

Accepted 28.07.2020

Published online 31.07.2020

Corresponding Author:

Kennedy Onyelowe

adaugofavour3@gmail.com

(C) 2020 The Authors. This article is licensed under a Creative Commons Attribution 4.0 License @) (1)
Abstract. Cardiovascular Disease (CVD), which is also known as Heart Disease has been the most common cause of death among diabetic and sickle cell anemia (SCA) patients around the world. CVD includes coronary artery disease, stroke, and peripheral artery disease while SCA includes hemolysis. These are the main types of CVD, which is similar to hypertension because of the common risk factors they have, such as obesity, arterial remodeling, abnormal cholesterol levels, etc. Diabetes and SCA belong to the largest health emergencies of the 21 st century. With the high rate of people with diabetes and SCA, the rate of Cardiovascular Disease increases rapidly. The rates of CVD in high-income countries generally have low CVD because of the monitoring systems for noncommunicable diseases like CVD. However, the appearance of the novel COVID-19/SARS-CoV-2 has changed the narrative. With COVID-19/SARSCoV-2 attacking more on people with previous health conditions associated to cardiovascular conditions, the backbone of high-income nations is broken and those leaving with high immune conditions are at less risk whether in the high income or low-income environments. The objective of this research is to review the Cardiovascular Disease conditions to the novel COVID-19/SARS-CoV-2 in Diabetic and SCA patients. This is due to the prevalence of this medical situation in the developing world or low income and densely populated countries.

Keywords: Coronavirus (SARS-CoV-2); Cardiovascular Conditions; SCA Patients; Diabetic Patients; Remdesivir; Aged Patients; Intravascular Coagulation; Biomedicine.

\section{INTRODUCTION}

Cardiovascular Disease (CVD) conditions refer to conditions that involve blocked blood vessels that can lead to a heart attack, chest pain, or stroke [1, 2, 3]. They include numerous problems which are related to a process called atherosclerosis [1]. Atherosclerosis is a condition that develops when a substance called plaque builds up in the walls of the arteries [3]. This can cause a heart attack or stroke. Research results in recent years have shown a connection between cardiovascular disorders in relation to the current novel COVID-19 medically caused by Severe Acute Respiratory Syndrome (SARS-CoV-2), sickle cell anemia and diabetes mellitus conditions $[4,5,6]$. Diabetes is a medical condition caused by a lack of insulin that makes its patients produce a lot of urine and feel very thirsty [7]. This by medical extension is caused by the inability of the pancreas to produce insulin due to certain health conditions [8]. There are some rampaging effects of diabetes in developing countries, for example, Nigeria includes blindness, kidney failure, heart attacks, stroke and lower limb amputation. The most common cause of cardiovascular conditions in diabetic patients is the hardening of the coronary arteries or atherosclerosis, which increases cholesterol in the blood vessels that supply oxygen and nutrition to the heart. Some examples of CVD include [9]:

1. Atrial Fibrillation which refers to the malfunction of the hearts internal electrical system which 
affects the rate and rhythm of the heart. It generally abbreviated as AF or AFib. Its causes include dysfunction of the sinus node, coronary artery disease, high blood pressure and rheumatic heart disease.

2. Coronary Heart Disease which occurs when a substance called plaque builds up in the walls of the arteries. A plaque makes it more difficult for the flow of blood to the heart.

3. Stroke which is defined as the interruption of blood flow to the brain, that leads to altered brain function, paralysis, slurred speech. The blockage in a blood vessel that carries blood to the brain is known as ischemic. Some signs include weakness of the face, arms or legs, having trouble speaking, trouble to see in one or both eyes, dizziness, loss of coordination.

4. High blood pressure refers to the pressure of blood against the walls of the arteries. When the presence of blood is above the normal range, it is said to be high which boosts the heart's workload and putting a person at very great risk of CVD. In $2015,41.5 \%$ which is about 102.7 million people from the U.S. had at least one CVD condition. CVD in the U.S. leads to the following statistical and bioinformatic data; High blood pressure about 96.1 million, Stroke about 7.5 million, Coronary Heart Disease about 16.8 million, and Atrial Fibrillation about 5.2 million. The case in Nigeria is as follows; High blood pressure about 11.4 million, Stroke about 1.14 million, Coronary Heart Disease about 86.1 million and Atrial Fibrillation about 15.9 million. In Asia, where the most severe data was capture is as follows; High blood pressure about 300 million, Stroke about 200 million, Coronal Heart Disease about 1.4 million and Atrial Fibrillation about 72 million people. It can be observed why more people are dying daily in Asia and Europe due to the novel coronavirus outbreak across the globe.

However, recent development and findings have shown that the primary cause of death in COVID$19 /$ SARS-CoV-2 patients is thrombosis and not what has been popularly and erroneously observed by experts $[8,10,11]$. It has been proven that around the world, COVID-19/SARS-CoV-2 is being attacked wrongly due to serious pathophysiological diagnosis error [10]. That error has occurred due to diagnostic errors and autopsies have proven that COVID-19/SARS-CoV-2 cause the dissemination of intravascular coagulation of the body transport system [10]. And this result, the research also proposed that the way to fight it by antibiotics, antiviral, anti-inflammatory and anticoagulants in a combination of pain relief medications. Further, home remedies have been suggested by researchers who are struggling in this effort for the use of $500 \mathrm{mg}$ aspirins dissolved in lemon juice boiled with honey and administered hot [11]. The results of this clinical trial have been impressive [12]. Italian medical research in pathology has shown that COVID19/SARS-CoV-2 patients don't die of pneumonia rather of an inflammatory storm that creates an endothelial vascular thrombosis in victims [13, 14]. In disseminated intravascular coagulation, the lung is the most affected as it inflames more than any other air transport mechanism in the body. Hence patients with preconditions of associated cardiovascular diseases like diabetes and SCA are of primary concern to this review work.

\section{RESULTS}

The world is currently gripped by the coronavirus which is caused by Severe Acute Respiratory Syndrome (SARS-CoV-2). According to research findings $[15,16,17]$, when a person is infected by this virus it induces an innate immune response when intracellular double-standard viral RNA is detected. Research has shown that COVID-19 can be divided into four stages which are, early infection, pulmonary phase, hyper inflammation, and recovery [17]. In SARS-CoV-2 the response of the body depends on the viral load of the body [18, $19,20]$. According to the results of findings [21, $22,23]$, people with type 2 diabetes (T2D) are at greater risk of breakdown if they are infected with SARS-CoV-2. This is due to the inhibited organ response they suffer when affected with severe respiratory conditions that burden the cardiovascular disposition of the patients [22]. The novel coronavirus has mutated into at least thirty strains wherein nineteen were never seen before [16]. According to research, eleven different patients suffering from COVID-19 were selected and tested, it was identified that more than thirty different mutations of the virus were discovered $[24,25]$. With so many mutations, it may be difficult to produce a vaccine that could be effective in preventing SARS-CoV-2 within the nearest possible frame of time. SARS-CoV-2 has been classified as an acute respiratory infection which causes multiorgan failure and death and these badly affects patients with somewhat terminal health conditions like diabetes and sickle cell anemia [24]. Those who suffer from deaths in 
COVID-19 are the elderly that are 80 years old and older 81, 89 and 90 [25]. According to [27], Israeli scientists have developed what is called monoclonal antibody from a recovered cell that neutralizes SARS-CoV-2 in hours. This antibody according to research is monoclonal, novel, refined and contains a low proportion of harmful proteins and it was specifically tested on aggressive COVID-19/SARS-CoV-2. According to [28], pangolins may be a novel key to the treatment of COVID-19/SARS-CoV-2, research has shown. This important discovery was made at the Medical University of Vienna, Austria on the immune response of the novel COVID-19/SARS-CoV-2 in pangolins. The research showed the genetic difference between the pangolins and other mammals and why the pangolins tolerate the virus thereby pointing the compass to the cure that lies ahead in the pangolins. Also, according to [29] in a previous research position, ivermectin was proposed as a cure for COVID-19/SARS-CoV-2 as it suppresses the viral load of the virus in 48 hours according to the research. Ivermectin is an antiparasitic drug that can reduce the virus in 48 hours. This drug has also been used against other viruses, such as zika, influenzas, and human immunodeficiency virus (HIV). It has been used to treat parasitic conditions like head lice, scabies and rosacea. This drug was developed in 1975 and has been of use around the world in the early '80s. But in a subsequent position according to [30], it has suggested that the approved dose of ivermectin according to Angela alone isn't useful in treating the virus, COVID-19/SARS-CoV-2. Also, Sally Robertson in a different position [31] has submitted that Joanne Lemieux and colleagues say the dipeptide-based protease inhibitor, GC376, and its analogue, GC373, should be fast-forwarded for testing in clinical trials of COVID-19. Accordingly, the discoveries have been considered as follows; "They are strong drug candidates for the treatment of human coronavirus infections because they have already been successful in animals (cats)," writes the team. "The work here lays the framework for their use in human trials for the treatment of COVID-19."

\section{SARS-CoV-2 to Cardiovascular Disease Conditions in Diabetic Patients}

Researchers have observed that a large proportion of patients with COVID-19 have underlying Cardiovascular Disease (CVD) conditions or Car- diac risk factor [32]. These include hypertension, diabetes mellitus, cardiovascular disease and heart failure. This viral infection can lead to acute coronary syndromes, arrhythmias, and heart failure and even death [33]. Cardiovascular conditions seem to be a threat in patients with SARSCoV-2 [34]. SARS-CoV-2 is associated with cardiac dysfunction [35]. Many patients with COVID19 were diagnosed as having an acute myocardial injury leading to cardiovascular trepidation [36]. Control of blood glucose has proven to be an effective way to manage, arrest or remove COVID-19 in diabetic patients [37]. Diabetic patients with well-controlled blood sugar and SARS-CoV-2 are less likely to die than those with poorly controlled blood-sugar according to research results [37]. According to research and findings, it has been said that Middle East Respiratory Syndrome-related coronavirus (MERS$\mathrm{CoV}$ ) can cause acute myocarditis and heart failure and even death [38]. In patients with MERSCoV infection and symptoms, $50 \%$ had hypertension and diabetes, up to $30 \%$ had heart disease therefore patients with CVD result in more portion of deaths from COVID-19 [36]. One point that should be noted is that the use of antiviral drugs can cause cardiac insufficiency, arrhythmia or even other cardiovascular disorders [35]. Therefore, the use of antiviral drugs must be closely monitored because of the high risk of cardiac toxicity and eventual complication arising therefrom.

\section{SARS-CoV-2 to Cardiovascular Disease (CVD) Conditions in Patients Living with Sickle Cell Anemia (SCA)}

Sickle cell disease (SCD) has been affecting 100,000 individuals in the united states, millions globally and especially the underdeveloped countries where health management is very poor [39]. Researchers have said that COVID-19 infection might keep coming back due to genetic recombination, so close contact with the medical team is required for individuals living with SCA [40]. These individuals suffer both acute and chronic complications that include cell pain, fever, and Acute Chest Syndrome (ASC) [39, 41]. ASC include chest pain, cough, fever and hypoxia [42]. During this pandemic, patients suffering from SCA should be on routine clinical care, reduce visits to the emergency departments and hospitals and should manage pain at home with oral medications [43]. SCD patients should con- 
tact the doctors or caregivers via telephone, texting or video conference to prevent the risk of contracting SARS-COV-2 [39]. Close monitoring for signs of Acute Chest Syndrome (ASC) is necessary when treating COVID-19 in these patients, because Sickle Cell Anemia (SCA) patients may sometimes have undiagnosed pulmonary hypertension, which could be a threat to COVID-19 management $[39,43]$. Donation of blood should be encouraged for patients living with SCA [39].

\section{Recent Developments in SARS/CoV-2 (COVID-19) Treatment}

There have been clinical trials going on recently in the treatment of SARS/COVID-19 around the world [27, 29, 30]. A set of such cases are the "Remdesivir" and the "Placebo" drugs being clinically studied for the treatment of the virus monitored by a combined effort of National Institute of Allergy and Infectious Diseases (NIAID) and Gilead Sciences, Incorporated under what is considered an Adaptive COVID-19 Treatment Trial (ACTT) [44]. These multiple ongoing clinical trials are being supported under investigational studies to evaluate the safety and efficacy of Remdesivir and Placebo as a potential cure for SARS-CoV-2 (COVID-19). Also, at the University of Nebraska Medical Center (UNMC) in Omaha, in the midwestern United States, a randomized clinical investigational trial is ongoing to evaluate the safety and efficacy of the antiviral Remdesivir on hospitalized adults diagnosed with COVID-19. So far, clinical trials show that Remdesivir accelerates faster recovery within a median time of 11 days from advanced COVID-19 against the median recovery time of 15 days for Placebo trials [44]. Secondly, the COVID-19 organic purported to have been discovered naturally as a herbal drink and endorsed by the Madagascar leadership. However, it is undergoing laboratory examinations and trials to understudy its safety and efficacy in the treatment of COVID-19 in Nigeria. There has also been a composite combination of turmeric-ginger-garlic tea but these have no scientific backing and certification on their safety and cure potentials. Vaccination is eminent if the rate at which this virus is spreading from index to community and phase two resulting from the ease in lockdown conditions in countries around the world.

\section{CONCLUSION}

The novel COVID-19/SARS-CoV-2 to cardiovascular conditions in diabetic and SCA patients has been reviewed using different related literature and it can be concluded that the cause of death in COVID-19/SARS-CoV-2 patients is intravascular coagulation. This is a cardiovascular condition which can also be associated with the major cause of death in diabetic and SCA patients. It has been posited that care must be taken in managing the health conditions of diabetic and SCA patients whether aged or young because of the close clinical association they have with the COVID-19/SARS-CoV-2 currently ravaging the world. It has also been proposed among other cure discovery efforts made by researchers, that the way to fight it is by antibiotics, antiviral, antiinflammatory and anticoagulants in a combination of pain relief medications. There have been clinical trials to evaluate the safety and efficacy of Remdesivir and Placebo as potential cures for SARS/CoV-2 (COVID-19), meanwhile, this has not been tried on the selected patients under investigation in this work. However, Remdesivir has been authorized in the United States and other ally countries for use under an Emergency Use Authorization (EUA), which allows its application in the treatment of suspected or laboratoryconfirmed patients. Furthermore, home remedies have been suggested by researchers who are struggling in this effort, for the use of $500 \mathrm{mg}$ aspirins dissolved in lemon juice boiled with honey and administered hot.

\section{ACKNOWLEDGEMENT}

The time and effort put by my mentor (my Dad), his friends, my Head of Department, Dr Celestine Afiukwa and my lecturer, Dr Stanley Onuoha of the Department of Biotechnology, Faculty of Science, Ebonyi State University are acknowledged and appreciated.

\section{CONFLICT OF INTEREST}

The authors declare no conflict of interest in this work. 


\section{REFERENCES}

1. Sakamoto, Y., Nishiyama, Y., Iwasaki, Y., Daida, H., Toyoda, K., Kitagawa, K., ... Shimizu, W. (2019). Design and rationale of the STroke secondary prevention with catheter ABLation and EDoxaban clinical trial in patients with non-valvular atrial fibrillation: The STABLED study. Journal of Cardiology, 74(6), 539-542. doi: 10.1016/j.jjcc.2019.06.002

2. Mukadas, A. O., \& Misbau, U. (2009). Incidence and Patters of Cardiovascular Disease in North Western Nigeria. Nigerian Medical Journal, 50(3), 55-57.

3. Coccheri, S. (2007). Approaches to Prevention of Cardiovascular Complications and Events in Diabetes Mellitus. Drugs, 67(7), 997-1026. doi: 10.2165/00003495-200767070-00005

4. Packer, M. (2017). Heart Failure: The Most Important, Preventable, and Treatable Cardiovascular Complication of Type 2 Diabetes. Diabetes Care, 41(1), 11-13. doi: 10.2337/dci17-0052

5. Cavan, D., Harding, J., Linnenkamp, U., Makaroff, L., Magliano, D., Ogurtsova, K., \& Shaw, J. (2016). Diabetes and cardiovascular disease. International Diabetes Federation. Retrieved from https://www.idf.org/our-activities/advocacy-awareness/resourcesand-tools/90:diabetes-and-cardiovascular-disease-report.html

6. Fornell, D. (2020). The Cardiac Implications of Novel Coronavirus. American College of Cardiology issues a clinical bulletin to update cardiologists about cardiovascular impacts of the COVID-19. Diagnostic and Interventional Cardiology. Retrieved from https://www.dicardiology.com/article/cardiac-implications-novel-coronavirus

7. Ferrario, C. M., Ahmad, S., \& Groban, L. (2020). Mechanisms by which angiotensin-receptor blockers increase ACE2 levels. Nature Reviews Cardiology, 17(6), 378-378. doi: 10.1038/s41569-0200387-7

8. Ives, J. (2020, May 4). Blood sugar control is key for people with type 2 diabetes and COVID19. Retrieved from https://www.news-medical.net/news/20200504/Blood-sugar-control-is-keyfor-people-with-type-2-diabetes-and-COVID-19.aspx

9. Rosano, G., Vitale, C., Seferovic, P. (2017). Heart failure in patients with diabetes mellitus. Cardiac Failure Review, 3(1), 52-55.

10. Sanchez, E. (2020, March 24). What heart patients should know about coronavirus. Retrieved from https://www.heart.org/en/news/2020/02/27/what-heart-patients-should-know-aboutcoronavirus

11. Laguipo, A. (2020, April 28). COVID-19 could be causing potentially fatal inflammatory disease in children, doctors warn. Retrieved from https://www.news-

medical.net/news/20200428/COVID-19-could-be-causing-potentially-fatal-inflammatorydisease-in-children-doctors-warn.aspx

12. Laguipo, A. (2020). Asymptomatic carriers transmit SARS-CoV-2 with normal breathing. Retrieved from https://www.news-medical.net/news/20200504/Asymptomatic-carriers-transmit-SARSCoV-2-with-normal-breathing.aspx

13. American Heart Association. (2020). Oxygenation and Ventilation of COVID-19 Patients. Module 4: Ventilation Management. Retrieved from https://cpr.heart.org/-/media/cprfiles/resources/covid-19-resources-for-cpr-training/oxygenation-and-ventilation-of-covid-19patients/ovcovid_mod4_vntmgmt_200401_ed.pdf

14. American Heart Association. (2020). Oxygenation and Ventilation of COVID-19 Patients. Module 1: Noninvasive Support Overview. Retrieved from https://www.aaup.edu/sites/default/files/OVCOVID_Mod1_Noninvasive_FIN_0.pdf

15. Sickle Cell Disease. Association of America (2020, May 27). Sickle cell disease and COVID-19: Provider Advisory. Retrieved from https://www.sicklecelldisease.org/2020/03/18/sickle-celldisease-and-covid-19-provider-directory 
16. Hu, B., Ge, X., Wang, L.-F., \& Shi, Z. (2015). Bat origin of human coronaviruses. Virology Journal, 12(1). doi: 10.1186/s12985-015-0422-1

17. Galevitz, Ph. (2020, May 8). Looking for a path to reopen, employers weigh COVID testing of workers. Retrieved from https://khn.org/news/looking-for-a-path-to-reopen-employers-weigh-covidtesting-of-workers

18. Mandal, A. (2020, March 29). COVID-19 and its effects on the cardiovascular system. Retrieved from https://www.news-medical.net/news/20200329/COVID-19-and-its-effects-on-thecardiovascular-system.aspx

19. Thomas, L. (2020, May 5). Hydroxychloroquine shown to slow recovery for COVID-19 patients. Retrieved from https://www.news-medical.net/news/20200505/Hydroxychloroquine-shownto-slow-recovery-for-COVID-19-patients.aspx

20. Thomas, L. (2020). Research detects a more dangerous SARS-CoV-2 Mutation. Retrieved from https://www.news-medical.net/news/20200504/Research-detects-a-more-dangerous-SARSCoV-2-mutation.aspx

21. Petrie, J. R., Guzik, T. J., \& Touyz, R. M. (2018). Diabetes, Hypertension, and Cardiovascular Disease: Clinical Insights and Vascular Mechanisms. Canadian Journal of Cardiology, 34(5), 575-584. doi: 10.1016/j.cjca.2017.12.005

22. Shimizu, I., \& Minamino, T. (2019). Cellular senescence in cardiac diseases. Journal of Cardiology, 74(4), 313-319. doi: 10.1016/j.jjcc.2019.05.002

23. Okura, K., Maeno, K., Okura, S., Takemori, H., Toya, D., Tanaka, N., \& Miyayama, S. (2015). Pericardial fat volume is an independent risk factor for the severity of coronary artery disease in patients with preserved ejection fraction. Journal of Cardiology, 65(1), 37-41. doi:

10.1016/j.jjcc.2014.03.015

24. Ghani, A. C., Donnelly, C. A., Cox, D. R., Griffin, J. T., Fraser, C., Lam, T. H., ... Leung, G. M. (2005). Methods for Estimating the Case Fatality Ratio for a Novel, Emerging Infectious Disease. American Journal of Epidemiology, 162(5), 479-486. doi: 10.1093/aje/kwi230

25. Clerkin, K. J., Fried, J. A., Raikhelkar, J., Sayer, G., Griffin, J. M., Masoumi, A., ... Uriel, N. (2020). COVID19 and Cardiovascular Disease. Circulation, 141(20), 1648-1655. doi:

10.1161/circulationaha.120.046941

26. Ma, S., Zhang, J., Zeng, M., Yun, Q., Guo, W., Zheng, Y., ... Yang, Z. (2020). Epidemiological parameters of coronavirus disease 2019: a pooled analysis of publicly reported individual data of 1155 cases from seven countries. doi: 10.1101/2020.03.21.20040329

27. Laguipo, A. (2020, May 5). Israeli scientists discover monoclonal antibody that neutralizes SARS-CoV2. Retrieved from https://www.news-medical.net/news/20200505/Israeli-scientists-discovermonoclonal-antibody-that-neutralizes-SARS-CoV-

2.aspx\#: :text=Now\%2C\%20in\%20a\%20significant\%20medical,outside\%20of\%20a\%20living \%20organism

28. Robertson, S. (2020, May 8). Pangolins may be the key to new COVID-19 Treatments. Retrieved from https://www.news-medical.net/news/20200508/Pangolins-may-be-the-key-to-new-COVID19-treatments.aspx

29. Laguipo, A. (2020, April 6). Antiparasitic drug Ivermectin kills coronavirus in 48 hours. Retrieved from https://www.news-medical.net/news/20200406/Antiparasitic-drug-Ivermectin-killscoronavirus-in-48-hours.aspx

30. Thomas, T. (2020, April 27). The approved dose of ivermectin alone not useful in treating COVID-19. Retrieved from https://www.news-medical.net/news/20200427/Ivermectin-alone-not-usefulin-treating-COVID-19.aspx 
31. Robertson, S. (2020, May 5). Cat coronavirus drug shows promise for treatment of COVID-19. Retrieved from https://www.news-medical.net/news/20200505/Cat-coronavirus-drug-showspromise-for-treatment-of-COVID-19.aspx

32. Mandal, A. (2020, May 25). What happens when COVID-19 and cardiovascular diseases mix? Retrieved from https://www.news-medical.net/news/20200325/What-happens-when-COVID19-and-cardiovascular-diseases-mix.aspx

33. Mourad, J.-J., \& Levy, B. I. (2020). Interaction between RAAS inhibitors and ACE2 in the context of COVID-19. Nature Reviews Cardiology, 17(5), 313-313. doi: 10.1038/s41569-020-0368-X

34. Kowalik, M. M., Trzonkowski, P., Łasińska-Kowara, M., Mital, A., Smiatacz, T., \& Jaguszewski, M. (2020). COVID-19 - Toward a comprehensive understanding of the disease. Cardiology Journal, 27(2), 99-114. doi: 10.5603/cj.a2020.0065

35. Laguipo, A. (2020, April 22). Coronavirus has mutated into at least 30 Strains. Retrieved from https://www.news-medical.net/news/20200422/Coronavirus-has-mutated-into-at-least-30strains.aspx\#: :text=Now\%2C\%20a\%20new\%20study\%20reveals, different\%20parts\%20of\% 20the\%20world.

36. Xiong, T.-Y., Redwood, S., Prendergast, B., \& Chen, M. (2020). Coronaviruses and the cardiovascular system: acute and long-term implications. European Heart Journal, 41(19), 1798-1800. doi: 10.1093/eurheartj/ehaa231

37. Zheng, Y.-Y., Ma, Y.-T., Zhang, J.-Y., \& Xie, X. (2020). COVID-19 and the cardiovascular system. Nature Reviews Cardiology, 17(5), 259-260. doi: 10.1038/s41569-020-0360-5

38. Ohm, J., Hjemdahl, P., Skoglund, P. H., Discacciati, A., Sundström, J., Hambraeus, K., ... Svensson, P. (2019). Lipid levels achieved after a first myocardial infarction and the prediction of recurrent atherosclerotic cardiovascular disease. International Journal of Cardiology, 296, 1-7. doi: 10.1016/j.ijcard.2019.07.001

39. Sickle Cell Disease Association of America. (2020, April 1). Health Alert for People with Sickle Cell Disease and their Caregivers. Retrieved from https://www.sicklecelldisease.org/files/sites/181/2020/03/MARAC-SCDAA-Patient-CaregiverAdvisory-AFRO-VERSION.pdf

40. Yilgwan, C., Hyacinth, H., Ige, O., Abok, I., Yilgwan, G., John, C., ... Bode-Thomas, F. (2017). Cardiovascular disease risk profile in Nigerian school children. Sahel Medical Journal, 20(4), 143. doi: 10.4103/1118-8561.230260

41. Hiebert, J. B., Vacek, J., Shah, Z., Rahman, F., \& Pierce, J. D. (2019). Use of speckle tracking to assess heart failure with preserved ejection fraction. Journal of Cardiology, 74(5), 397-402. doi: 10.1016/j.jjcc.2019.06.004

42. Yakabe, D., Aso, A., Araki, M., Murasato, Y., \& Nakamura, T. (2020). Efficacy of hybrid therapy using prior administration of bepridil hydrochloride and cryoballoon ablation in patients with persistent atrial fibrillation. Journal of Cardiology, 75(4), 360-367. doi: 10.1016/j.jjcc.2019.08.017

43. Rahman, S., Majumder, M. A. A., Kabir, R., Haque, M., Gupta, S., Arafat, S. M. Y., ... Dalvi, P. (2017). Cardiovascular Disease and Diabetes: Two Sides of the Same Coin! Recent Trends in Cardiovascular Risks. doi: 10.5772/intechopen.69038

44. National Institute of Health. (2020, April 29). NIH clinical trial shows Remdesivir accelerates recovery from advanced COVID-19. Retrieved from https://www.nih.gov/news-events/newsreleases/nih-clinical-trial-shows-remdesivir-accelerates-recovery-advanced-covid-19 